\title{
Rate Splitting Approach Under PSK signaling Using Constructive Interference Precoding Technique
}

\author{
Abdelhamid Salem and Christos Masouros \\ Department of Electronic and Electrical Engineering, \\ University College London, London, UK \\ emails: \{a.salem, c.masouros\}@ucl.ac.uk
}

\begin{abstract}
Rate-Splitting (RS) approach has been proposed recently to enhance the performance of multi-user multiple-input multiple-output (MU-MIMO) systems. In RS a user message is split into common and private parts, where the common message can be decoded by all users, while the private one can be decoded only by the intended user. In addition, constructive interference (CI) precoding technique has shown to provide significant performance benefits in different multi-user scenarios. In this paper we propose employing the CI concept to further enhance the sum-rate achieved by RS approach in MU-MIMO systems under a phase-shift keying (PSK) input alphabet. In light of this and in order to provide fair comparison, new analytical expressions for the average sum-rate are derived for two precoding techniques of the private messages, namely, 1) CI precoding technique, 2) zero forcing (ZF) precoding technique. In addition, the conventional transmission, without using RS (NoRS) is also studied in this paper. Monte-Carlo simulations are provided throughout to confirm the analysis. The results in this work validate the significant sum-rate gain of $\mathrm{RS}$ with $\mathrm{CI}$ over the conventional RS with ZF technique.
\end{abstract}

Index Terms-Rate splitting, zero forcing, constructive interference, phase-shift keying signaling.

\section{INTRODUCTION}

$\mathbf{T}$ HE recent years have witnessed the widespread application of multi-user multiple-input multiple-output (MUMIMO) systems, due to their reliability high spectral efficiency and. However, the advantages of MU-MIMO systems are often impacted by interference in practical communication networks. Consequently, considerable amount of researches have focused on improving the performance of MU-MIMO systems [1]. In this regard, Rate-Splitting (RS) approach was recently proposed and investigated in different scenarios [2]-[5]. In RS scheme the users' messages are split into a common message and private messages. The common message is considered to be decoded by all the users, while the private messages can be decoded only by the intended user. At the reception, each user firstly decode the common message, and then uses successive interference cancellation (SIC) technique to decode its own private message. In [3] the authors studied the average sum-rate in two scenarios, when the common message is transmitted via a space and space-time design. In [4] it was proved that the RS strategy outperforms the conventional, without rate splitting (NoRS), in interference limited systems. RS technique was proposed in [5] as an efficient solution to tackle the saturation in MIMO cooperative systems. Moreover, constructive interference $(\mathrm{CI})$ precoding technique has been proposed recently to enhance the performance of MU-MIMO systems [6], [7]. In contrast to the conventional techniques where the knowledge of the interference is used to cancel it, the main idea of the $\mathrm{CI}$ is to exploit the interference to improve the system performance [6], [7]. With the knowledge of the users' data symbols and the channel state information (CSI), the interference can be classified into constructive and destructive. The interference is considered to be constructive to the transmitted signal if it moves the received symbols away from the decision thresholds of the constellation towards the direction of the desired symbol. Consequently, transmit precoding can be designed to make the interference constructive to the desired symbol. This line of work was introduced in [6], where the CI precoding scheme of phase-shift keying (PSK)-based MIMO systems has been proposed. The work in [7] applied the CI concept to massive multi-input multioutput (M-MIMO) systems. In [8] CI precoding was applied in wireless power transfer scenario to minimize the transmit power while guaranteeing the quality of service (QoS) and the energy harvesting constraints. Very recently, in [9] the authors derived closed-form expression for CI exploitation precoding in MU-MIMO systems. This closed-form of the CI precoder has paved the way for the development of communication theoretic analysis of the benefits of CI technique.

Accordingly, in this paper we employ the CI precoding technique to further enhance the sum-rate achieved by RS scheme in MU-MIMO systems under a PSK input alphabet. In this regard, new analytical expressions for the average sumrate are derived for two precoding techniques of the private messages, namely, 1) CI precoding technique, 2) zero forcing $(\mathrm{ZF})$ precoding technique. Additionally, the conventional transmission, NoRS, is also studied in this paper. Furthermore, we provide Monte-Carlo simulations to confirm our analysis, and the impact of different system parameters on the achievable sum-rate are investigated. The results in this work show clearly that, the sum-rate of RS with CI outperforms the sum rate of RS with $\mathrm{ZF}$ and NoRS transmission techniques.

Notations: $h, \mathbf{h}$, and $\mathbf{H}$ denote a scalar, a vector and a matrix, respectively. $(\cdot)^{H},(\cdot)^{T}$ and $\operatorname{diag}($.$) denote conjugate$ transposition, transposition and diagonal of a matrix, respectively. $\mathcal{E}[$.$] denotes average operation. [\mathbf{h}]_{k}$ denotes the $k^{t h}$ element in $\mathbf{h},|$.$| denotes the absolute value, , and \|.\|^{2}$ denotes the second norm. $\mathbb{C}^{K \times N}$ represents an $\mathrm{K} \times \mathrm{N}$ matrix, and $\mathbf{I}$ denotes the identity matrix. 


\section{SySTEM MODEL}

We consider a MU-MIMO system, in which $N$-antennas base station (BS) node communicating with $K$-single antenna users in down-link scenario using RS technique. The channels in this system are modeled as independent identically distributed (i.i.d) Rayleigh fading channels. The channel matrix between the BS and the users is denoted by $\mathbf{H} \in \mathbb{C}^{K \times N}$, which can be expressed as $\mathbf{H}=\mathbf{D}^{1 / 2} \mathrm{G}$ where $\mathrm{G} \in \mathbb{C}^{K \times N}$ contains i.i.d $\mathcal{C N}(0,1)$ entries represent small-scale fading coefficients and $\mathbf{D} \in \mathbb{C}^{K \times K}$ is a diagonal matrix represents the path-loss attenuation with $[\mathbf{D}]_{k k}=d_{k}^{-m}$, where $d_{k}$ is the distance between the BS and the $k^{t h}$ user, and $m$ is the path loss exponent. It is also assumed that the signal is equiprobably drawn from a $M$-PSK constellation.

In $\mathrm{RS}$, the signal for a user is split into a common part and a private part, where the common part can be decoded by the all users with zero error probability, while the private one is detected only by the intended user. The common and the private symbols are denoted by $x_{c}$ and $x_{k}, k \in[1,2, \ldots, K]$, respectively. Hence, $K+1$ symbols of a given channel use can be grouped in a vector $\mathbf{x}=\left[x_{c}, x_{1}, \ldots, x_{K}\right]^{T} \in \mathbb{C}^{N}$. Therefore, the transmitted signal can be mathematically expressed by [2], [3]

$$
\mathbf{s}=\mathbf{W} \mathbf{x}=\sqrt{P_{c}} \mathbf{w}_{c} x_{c}+\sum_{k=1}^{K} \sqrt{P_{p}} \mathbf{w}_{k} x_{k}
$$

where $\mathbf{W}=\left[\mathbf{w}_{c}, \mathbf{w}_{1}, \ldots . \mathbf{w}_{K}\right], \mathbf{w}_{c}$ denotes the common precoder of the common message and $\mathbf{w}_{k}$ is the $k^{t h}$ private precoder. In addition, $P_{c}$ is the power allocated to the common message and $P_{p}$ is the power allocated to the private message, where $P_{c}=(1-t) P$ and $P_{p}=\frac{t P}{K}, 0<t \leq 1$ and $P$ is the total power. The received signal at the $k^{t h}$ user in this system can be written as,

$y_{k}=\mathbf{h}_{k} \mathbf{W} \mathbf{x}+n_{k}=\sqrt{P_{c}} \mathbf{h}_{k} \mathbf{w}_{c} x_{c}+\sum_{k=1}^{K} \sqrt{P_{p}} \mathbf{h}_{k} \mathbf{w}_{k} x_{k}+n_{k}$

where $\mathbf{h}_{k}$ is the channel vector from the BS to user $k, n_{k}$ is the additive wight Gaussian noise (AWGN) at the $k^{\text {th }}$ user, $n_{k} \sim \mathcal{C N}\left(0, \sigma_{k}^{2}\right)$. At the user side, the common symbol is decoded firstly by treating the private messages as interference, and then each user decodes its own message after removing the common message using SIC technique. Therefore, the received private signal at the $k^{t h}$ user in this system can be written as,

$$
y_{k}^{p}==\sqrt{P_{p}} \mathbf{h}_{k} \mathbf{W}^{p} \mathbf{x}^{p}+n_{k}
$$

where $\mathbf{x}^{p}=\left[x_{1}, \ldots, x_{K}\right]^{T}$ and $\mathbf{W}^{p}=\left[\mathbf{w}_{1}, \ldots . \mathbf{w}_{K}\right]$. The sum rate in this scenario can be expressed by,

$$
R=R^{c}+\sum_{k=1}^{K} R_{k}^{p}
$$

where $R^{c}$ is the rate for the common part, $R^{c}=$ $\min \left(R_{1}^{c}, R_{2}^{c}, . ., R_{k}^{c}, . ., R_{K}^{c}\right), R_{k}^{c}$ is the rate for the common message at user $k$, and $R_{k}^{p}$ is the rate for the private part at the $k^{t h}$ user. In the following two precoding techniques are considered.

\section{MRT/CI}

In this scenario MRT technique is used for common message and CI precoding for the private messages. Therefore, the precoder for the common and the private messages can be written, respectively, as [2], [9]

$$
\begin{gathered}
\mathbf{w}_{c}=\sum_{i=1}^{K} \beta_{c} \mathbf{h}_{i}^{H} \\
\mathbf{W}_{C I}^{p}=\frac{1}{K} \beta_{p} \mathbf{H}^{H}\left(\mathbf{H} \mathbf{H}^{H}\right)^{-1} \operatorname{diag}\left\{\mathbf{V}^{-1} \mathbf{u}\right\},
\end{gathered}
$$

where $\beta_{c}$ and $\beta_{p}$ are the scaling factor to meet the transmit power constraint at the transmitter, while $\mathbf{V}=$ $\operatorname{diag}\left(\mathbf{x}^{p H}\right)\left(\mathbf{H H}^{H}\right)^{-1} \operatorname{diag}\left(\mathbf{x}^{p}\right)$ and $\mathbf{1}^{T} \mathbf{u}=1$. For simplicity and mathematical tractability but without loss of generality, the normalization constants $\beta_{c}$ and $\beta_{p}$ are designed to be constants and given by [1], [9], $\beta_{c}=\frac{1}{\sqrt{N \sum_{i=1}^{K} \varpi_{k}}}$ and $\beta_{p}=$ $\frac{1}{\sqrt{\mathbf{u}^{H} \operatorname{diag}\left(\mathbf{x}^{H}\right)^{-1} N \mathbf{\Sigma}(\operatorname{diag}(\mathbf{x}))^{-1} \mathbf{u}}}$, where $\Sigma$ is the covariance of the Wishart matrix $\left(\mathbf{H} \mathbf{H}^{H}\right)$ and $\varpi_{k}=d_{k}^{-m}$ [10].

\section{A. Rate for the Common Part}

Following the principles of CI, the rate for the common part at user $k$ under PSK signaling can be written as [11]-[13],

$$
\begin{gathered}
R_{k}^{c}=\log _{2} M-\frac{1}{M^{N}} \sum_{m=1}^{M^{N}} \underbrace{\mathcal{E}_{\mathbf{h}, n_{k}}\left\{\log _{2} \sum_{i=1}^{M^{N}} e^{\frac{-\left|\mathbf{h}_{k} \mathbf{w} \mathbf{x}_{m, i}+n_{k}\right|^{2}}{\sigma_{k}^{2}}}\right\}}_{\varphi} \\
+\frac{1}{M^{N-1}} \sum_{m=1}^{M^{N-1}} \underbrace{\mathcal{E}_{\mathbf{h}, n_{k}}\left\{\log _{2} \sum_{i=1}^{M^{N-1}} e^{\frac{-\left|\mathbf{h}_{k} \mathbf{w}_{C I}^{p} \mathbf{x}_{m, i}^{p}+n_{k}\right|^{2}}{\sigma_{k}^{2}}}\right\}}_{\psi},
\end{gathered}
$$

where $\mathbf{x}_{m, i}=\mathbf{x}_{m}-\mathbf{x}_{i}, \mathbf{x}_{m}$ and $\mathbf{x}_{i}$ contain symbols taken from the $M$ signal constellation. By invoking Jensen inequality, the first term $\varphi$ can be expressed by,

$$
\varphi \leq \log _{2} \sum_{i=1}^{M^{N}} \mathcal{E}_{\mathbf{h}, n_{k}}\left\{e^{\frac{-\left|\mathbf{h}_{k} \mathbf{W} \mathbf{x}_{m, i}+n_{k}\right|^{2}}{\sigma_{k}^{2}}}\right\}
$$

Since the noise $n_{k}$ has Gaussian distribution, the average over the noise can be derived as

$\mathcal{E}_{n_{k}}\left\{e^{\frac{-\left|\mathbf{h}_{k} \mathbf{W} \mathbf{x}_{m, i}+n_{k}\right|^{2}}{\sigma_{k}^{2}}}\right\}=\frac{1}{\pi \sigma^{2}} \int_{n_{k}} e^{-\frac{\left|\mathbf{h}_{k} \mathbf{W} \mathbf{x}_{m, i}+n_{k}\right|^{2}+\left|n_{k}\right|^{2}}{\sigma_{k}^{2}}} d n_{k}$.

$$
=e^{-\frac{\left|\mathbf{h}_{k} \mathbf{W} \mathbf{x}_{m, i}\right|^{2}}{2 \sigma_{k}^{2}}}
$$


Now, the average over the channel can be derived as

$$
\begin{gathered}
\mathcal{E}_{\mathbf{h}}\left\{e^{-\frac{\left|\mathbf{h}_{k} \mathbf{W} \mathbf{x}_{m, i}\right|^{2}}{2 \sigma_{k}^{2}}}\right\}= \\
\mathcal{E}_{\mathbf{h}}\left\{e^{-\frac{\left|\sqrt{P_{c}} \mathbf{h}_{k} \mathbf{w}_{c}\left[\mathbf{x}_{m, i}\right]_{1}+\sqrt{P_{p}} \mathbf{h}_{k} \mathbf{W}_{C I}^{p} \mathbf{x}_{m, i}^{p}\right|^{2}}{2 \sigma_{k}^{2}}}\right\}
\end{gathered}
$$

which can be written as in (12), where $\mathbf{a}_{k}$ is a $1 \times K$ vector all the elements of this vector are zeros except the $k^{t h}$ element is one.

Therefore, the first term $\varphi$ can be expressed as in (13), where $\xi=\frac{\beta_{p}}{K} \mathbf{a}_{k}\left(\operatorname{diag}\left(\mathbf{x}^{p H}\right)\right)^{-1}(\Sigma)\left(\operatorname{diag}\left(\mathbf{x}^{p}\right)\right)^{-1} \mathbf{u}$.

Now, we can simplify the last expression in (13) to,

$$
\varphi=\log _{2} \sum_{i=1}^{M^{N}} \mathcal{E}_{\mathbf{h}}\left\{e^{-\frac{P|\xi|^{2}\left\|\mathbf{h}_{k}\right\|^{2} \Psi_{m, i}}{2 \sigma_{k}^{2}}}\right\}
$$

when the term $\Psi_{m, i}$ in (13) can be approximated with high accuracy to $\Psi_{m, i}=\left|\frac{\sqrt{(1-t)} N \beta_{c}\left[\mathbf{x}_{m, i}\right]_{1}}{|\xi|}+\sqrt{t} N \beta_{p}\left[\mathbf{x}_{m, i}^{p}\right]_{k}\right|^{2}$. Since $\left\|\mathbf{h}_{k}\right\|^{2}$ has Gamma distribution, the average in (14) is the moment generating function (MGF) of the term, $\frac{P|\xi|^{2}\left\|\mathbf{h}_{k}\right\|^{2} \Psi_{m, i}}{2 \sigma_{k}^{2}}$ which can be found easily as

$$
\varphi=\log _{2} \sum_{i=1}^{M^{N}}\left(1+\frac{P|\xi|^{2} \varpi_{k} \Psi_{m, i}}{2 \sigma_{k}^{2}}\right)^{-N}
$$

For the second term, $\psi$, using Jensen inequality we can write

$$
\psi \leq \log _{2} \sum_{i=1}^{M^{N-1}} \mathcal{E}_{\mathbf{h}, n_{k}}\left\{e^{\frac{-\left|\sqrt{P_{p}} \mathbf{h}_{k} \mathbf{w}_{C I}^{p} \mathbf{x}_{m, i}^{p}+n_{k}\right|^{2}}{\sigma_{k}^{2}}}\right\}
$$

Similarly as in (9), since $n_{k}$ has Gaussian distribution, we can write $\psi$ as

$$
\psi=\log _{2} \sum_{i=1}^{M^{N-1}} \mathcal{E}_{\mathbf{h}}\left\{e^{-\frac{\left|\left(\frac{\sqrt{P_{p}} \beta_{p}}{K} a\left(\mathbf{H H}^{H}\right) b\left[\mathbf{x}_{m, i}^{p}\right]_{k}\right)\right|^{2}}{2 \sigma_{k}^{2}}}\right\}
$$

where $a=\mathbf{a}_{k}\left(\operatorname{diag}\left(\mathbf{x}^{p H}\right)\right)^{-1}$ and $b=\left(\operatorname{diag}\left(\mathbf{x}^{p}\right)\right)^{-1} \mathbf{u}$. It was shown that, $Y=\frac{\boldsymbol{a}\left(\mathbf{H H}^{H}\right) \boldsymbol{b}}{\mathbf{a} \Sigma \boldsymbol{b}}$ has a Gamma distribution [10]. Therefore we can rewrite (17) as

$$
\psi=\log _{2} \sum_{i=1}^{M^{N-1}} \mathcal{E}_{\mathbf{h}}\left\{e^{-\frac{\left|c Y\left[\mathbf{x}_{m, i}^{p}\right]_{k}\right|^{2}}{2 \sigma_{k}^{2}}}\right\}
$$

where $c=\frac{\sqrt{P t} \beta_{p} \mathbf{a} \Sigma \boldsymbol{b}}{K}$. Consequently, we can obtain, $\psi$, as in (19), where ${ }_{1} \mathrm{~F}_{1}$ is the Hypergeometric function.

\section{B. Rate for the Private Part}

Following the principles of CI, the rate for the private part at user $k$ under PSK signaling, using CI precoding technique can be written as [11]-[13],

$$
\begin{gathered}
R_{k}^{p}=\log _{2} M-\log _{2} e \\
-\frac{1}{M^{N}} \sum_{m=1}^{M^{N}} \underbrace{\mathcal{E}_{\mathbf{h}, n_{k}}\left\{\log _{2} \sum_{i=1}^{M^{N}} e^{\frac{-\left|\sqrt{P_{p}} \mathbf{h}_{k} \mathbf{w}_{C I}^{p} \mathbf{x}_{m, i}^{p}+n_{k}\right|^{2}}{\sigma_{k}^{2}}}\right\}}_{\psi}
\end{gathered}
$$

By using Jensen inequality, and following similar steps as in the previous sub-section we can find $\psi$ as in (19).

\section{MRT/ZF}

In this case we implement MRT technique for common signal and ZF precoding for the private messages. Therefore, the precoding for the common and the private messages can be written, respectively, as

$$
\begin{gathered}
\mathbf{w}_{c}=\sum_{i=1}^{K} \beta_{c} \mathbf{h}_{i}^{H} \\
\mathbf{W}_{Z F}^{p}=\beta_{p} \mathbf{H}^{H}\left(\mathbf{H} \mathbf{H}^{H}\right)^{-1},
\end{gathered}
$$

For comparison's sake and mathematical tractability, the normalization constants $\beta_{c}$ and $\beta_{p}$ are designed to be constants and given by [1], $\beta_{c}=\frac{1}{\sqrt{N \sum_{i=1}^{K} \varpi_{k}}}$ and $\beta_{p}=$ $\frac{\sqrt{\frac{1}{\mathrm{~s}^{H} \Sigma^{-1} \mathrm{~s}}} \Gamma\left(\frac{3}{2}-K+N\right)}{K \sqrt{K}(N-K) !}$, respectively.

\section{A. Rate for the Common Part}

The rate for the common part at user $k$ can be written as,

$$
\begin{gathered}
R_{k}^{c}=\underbrace{}_{\varphi} \underbrace{}_{\mathcal{E}_{\mathbf{h}, n_{k}}\left\{\log _{2} M\right.} \sum_{i=1}^{\left.M^{N} e^{\frac{-\left|\mathbf{h}_{k} \mathbf{W} \mathbf{x}_{m, i}+n_{k}\right|^{2}}{\sigma_{k}^{2}}}\right\}} \\
+\frac{1}{M^{N-1}} \sum_{m=1}^{M^{N-1}} \underbrace{\mathcal{E}_{\mathbf{h}, n_{k}}\left\{\log _{2} \sum_{i=1}^{M^{N-1}} e^{\frac{-\left|\mathbf{h}_{k} \mathbf{w}_{Z F}^{p} \mathbf{x}_{m, i}^{p}+n_{k}\right|^{2}}{\sigma_{k}^{2}}}\right\}}_{\psi},
\end{gathered}
$$

By using Jensen inequality, the first term $\varphi$ can be expressed as,

$$
\varphi \leq \log _{2} \sum_{i=1}^{M^{N}} \mathcal{E}_{\mathbf{h}, n_{k}}\left\{e^{\frac{-\left|\mathbf{h}_{k} \mathbf{W} \mathbf{x}_{m, i}+n_{k}\right|^{2}}{\sigma_{k}^{2}}}\right\}
$$

Since the noise $n_{k}$ has Gaussian distribution, using the integrals of exponential function in [14] the average over the noise can be derived as 


$$
\mathcal{E}_{\mathbf{h}}\left\{e^{-\frac{\left|\mathbf{h}_{k} \mathbf{W} \mathbf{x}_{m, i}\right|^{2}}{2 \sigma_{k}^{2}}}\right\}=\mathcal{E}_{\mathbf{h}}\left\{e^{-\frac{\left|\sqrt{P_{c} \beta_{c}}\left(\sum_{i=1}^{K} \mathbf{h}_{k} \mathbf{h}_{i}^{H}\right)\left[\mathbf{x}_{m, i}\right]_{1}+\left(\frac{\sqrt{P_{p}} \beta_{p}}{K} \mathbf{a}_{k}\left(\operatorname{diag}\left(\mathbf{x}^{p H}\right)\right)^{-1}\left(\mathbf{H H}^{H}\right)\left(\operatorname{diag}\left(\mathbf{x}^{p}\right)\right)^{-1} \mathbf{u}\left[\mathbf{x}_{m, i}^{p}\right]_{k}\right)\right|^{2}}{2 \sigma_{k}^{2}}}\right\} .
$$

$\varphi=\log _{2} \sum_{i=1}^{M^{N}} \mathcal{E}_{\mathbf{h}} e^{-\frac{P_{P|\xi|^{2}\left\|\mathbf{h}_{k}\right\|^{2}}}{\left.\overbrace{\frac{\sqrt{(1-t)} \beta_{c}\left(\sum_{i=1}^{K} \mathbf{h}_{k} \mathbf{h}_{i}^{H}\right)\left[\mathbf{x}_{m, i}\right]_{1}}{|\xi|\left\|\mathbf{h}_{k}\right\|}+\frac{\sqrt{t} \beta_{p} \mathbf{a}_{k}\left(\operatorname{diag}\left(\mathbf{x}^{p H}\right)\right)^{-1}\left(\mathbf{H H}^{H}\right)\left(\operatorname{diag}\left(\mathbf{x}^{p}\right)\right)^{-1} \mathbf{u}\left[\mathbf{x}_{m, i}^{p}\right]_{k}}{|\xi| K\left\|\mathbf{h}_{k}\right\|}}^{\Psi_{m, i}}\right|^{2}}}$

$$
\begin{aligned}
\psi= & \log _{2} \sum_{i=1}^{M^{N-1}}\left(\left(\frac{2^{\left(\frac{1}{2}(N-K-1)\right)} K^{(N-K+1)}\left|\left[\mathbf{x}_{m, i}^{p}\right]_{k}\right|^{-2+K-N}}{(N-K) !}\right)\left(\left(\frac{c^{2}}{\sigma_{k}^{2}}\right)^{\frac{1}{2}(K-N-1)}\right)\right) \\
& \times\left(\left(c^{2}\left|\left[\mathbf{x}_{m, i}^{p}\right]_{k}\right|\right) \Gamma\left(\frac{1}{2}(N-K+1)\right){ }_{1} \mathrm{~F}_{1}\left(\frac{1}{2}(N-K+1), \frac{1}{2}, \frac{K^{2} \sigma_{k}^{2}}{2 c^{2}\left|\left[\mathbf{x}_{m, i}^{p}\right]_{k}\right|^{2}}\right)\right. \\
& \left.\left.-\sqrt{2} K c \sigma_{k}^{2} \Gamma\left(\frac{1}{2}(N-K+2)\right){ }_{1} \mathrm{~F}_{1}\left(\frac{1}{2}(N-K+2), \frac{3}{2}, \frac{K^{2} \sigma_{k}^{2}}{2 c^{2}\left|\left[\mathbf{x}_{m, i}^{p}\right]_{k}\right|^{2}}\right)\right)\right)
\end{aligned}
$$

$$
\mathcal{E}_{n}\left\{e^{\frac{-\left|\mathbf{h}_{k} \mathbf{W} \mathbf{x}_{m, i}+n_{k}\right|^{2}}{\sigma_{k}^{2}}}\right\}=e^{-\frac{\left|\mathbf{h}_{k} \mathbf{W} \mathbf{x}_{m, i}\right|^{2}}{2 \sigma_{k}^{2}}} .
$$

Now, we can write $\varphi$ as

$$
\begin{gathered}
\varphi=\log _{2} \sum_{i=1}^{M^{N}} \mathcal{E}_{\mathbf{h}}\left\{e^{-\frac{\left|\mathbf{h}_{k} \mathbf{W} \mathbf{x}_{m, i}\right|^{2}}{2 \sigma_{k}^{2}}}\right\}, \\
=\log _{2} \sum_{i=1}^{M^{N}} \mathcal{E}_{\mathbf{h}}\left\{e^{-\frac{P\left|\sqrt{(1-t)} \beta_{c}\left(\sum_{i=1}^{K} \mathbf{h}_{k} \mathbf{h}_{i}^{H}\right)\left[\mathbf{x}_{m, i}\right]_{1}+\sqrt{t} \beta_{p}\left[\mathbf{x}_{m, i}^{p}\right]_{k}\right|^{2}}{2 \sigma_{k}^{2}}}\right\} .
\end{gathered}
$$

since the term $Y=\left(\sum_{i=1}^{K} \mathbf{h}_{k} \mathbf{h}_{i}^{H}\right)$ has Gamma distribution, .i.e., $Y \sim \Gamma(v, \theta)$, the average can be derived as,

$\varphi=\log _{2} \sum_{i=1}^{M^{N}} \sum_{r=1}^{n} \frac{\left(y_{r}\right)^{v-1} \mathrm{H}_{r}}{\Gamma(v)} e^{-\frac{P\left|\sqrt{(1-t)} \beta_{c} \theta y_{r}\left[\mathbf{x}_{m, i}\right]_{1}+\sqrt{t} \beta_{p}\left[\mathbf{x}_{m, i}^{p}\right]_{k}\right|^{2}}{2 \sigma_{k}^{2}}}$

where $y_{r}$ and $\mathrm{H}_{r}$ are the $r^{\text {th }}$ zero and the weighting factor of the Laguerre polynomials, respectively [14]. Similarly, for the second term $\psi$, using Jensen inequality and since the noise $n_{k}$ has Gaussian distribution, the average can be derived as

$$
\psi=\log _{2} \sum_{t=1}^{M^{N-1}} e^{-\frac{\left|\sqrt{t P} \beta_{p}\left[\mathbf{x}_{m, i}^{p}\right]_{k}\right|^{2}}{2 \sigma_{k}^{2}}}
$$

\section{B. Rate for the Private Part}

The rate for the private message at the $k^{\text {th }}$ user, under PSK signaling using ZF precoding technique can be written as [11][13],

$$
\begin{gathered}
R_{k}^{p}=\log _{2} M-\log _{2} e \\
-\frac{1}{M^{N}} \sum_{m=1}^{M^{N}} \underbrace{\mathcal{E}_{\mathbf{h}, n_{k}}\left\{\log _{2} \sum_{i=1}^{M^{N}} e^{\frac{-\left|\sqrt{P_{p}} \mathbf{h}_{k} \mathbf{W}_{Z F}^{p} x_{m, i}^{p}+n_{k}\right|^{2}}{\sigma_{k}^{2}}}\right\}}_{\psi} .
\end{gathered}
$$

By using Jensen inequality, and following similar steps as in the previous section we can find $\psi$ as in (28).

\section{Conventional Transmission NoRS}

The rate at the $k^{t h}$ user in conventional transmission without RS is expressed by

$$
R_{k}^{N o R S}=\log _{2} M-\log _{2} e
$$




$$
-\frac{1}{M^{N}} \sum_{m=1}^{M^{N}} \underbrace{\mathcal{E}_{\mathbf{h}, n_{k}}\left\{\log _{2} \sum_{i=1}^{M^{N}} e^{\frac{-\left|\sqrt{P} \mathbf{h}_{k} \mathbf{W} \mathbf{x}_{m, i}+n_{k}\right|^{2}}{\sigma_{k}^{2}}}\right\}}_{\psi}
$$

In $\mathrm{CI}$ case, the precoding matrix $\mathbf{W}$ is given in (6), and the expectation in (30) can be derived as in (19). On the other hand, in ZF scenario the precoding matrix $\mathbf{W}$ is given in (22), and then the expectation in (30) can be obtained as in (28).

\section{Power Allocation}

The optimal value of $t$ that achieves optimal sum-rate can be derived by maximizing (4). However, due to the complicated rate formula in the considered scenarios, we present here a sub-optimal solution as considered in [2]. In this solution, we allocate a fraction $t$ of the total power for the private messages to achieve same sum-rate as the conventional techniques with full power. Then, the remaining power is allocated for the common message. The sum-rate payoff of the RS scheme over the NoRS can be determined by, $\Delta R=R_{c}+\sum_{k=1}^{K}\left(R_{k}^{p}-R_{k}^{N o R S}\right)$. Consequently, the power ratio $t$ that achieves the superiority can be obtained by satisfying the equality, $R_{k}^{p}=R_{k}^{N o R S}$. Due to the complicated rate formula in finite alphabet scenarios, and to the paper length limitation, the best value of $t$ is obtained by using search algorithm techniques.

\section{Numerical Results}

In this section we present numerical results of the analytical expressions derived in this work. Monte-Carlo simulations are conducted, where the channel coefficients are randomly generated. The path loss exponent is $m=2.7$, assuming the users have same noise power, $\sigma^{2}$, and the total transmission power is $p$, the signal to noise ratio (SNR) is defined as SNR $=\frac{p}{\sigma^{2}}$.

In Fig. 1 we illustrate the sum-rate for the NoRS and RS with MRT-CI and MRT-ZF precoding techniques, subject to BPSK, and QPSK, when $N=3$, and $K=2$. Fig. 1a presents the sum-rate when the distances between the BS and the users are normalized to unit value, i.e, without the impact of the path-loss. To investigate the impact of the path-loss on the sum-rate, in Fig. 1b we plot the sum-rate when the users are uniformly distributed inside a circle area with a radius of $60 \mathrm{~m}$, and no user is closer to the BS than $1 \mathrm{~m}$ where the BS is located at the center of this area. The good agreement between the analytical and simulated results confirms the validity of the analysis introduced in this paper. Several observations can be noticed from this figure. Firstly, it is clear that the sum rate saturates at a certain SNR value, owing to the finite constellation. Secondly, the RS scheme enhances the sum-rate of the considered system and tackles the sum-rate saturation occurred in the communication systems with PSK signaling. In addition, it is evident that the CI precoding techniques outperforms the ZF technique in the all considered scenarios. Finally, comparing Fig. 1a and Fig. 1b, one can notice that, in general, increasing the distance always degrades the achievable

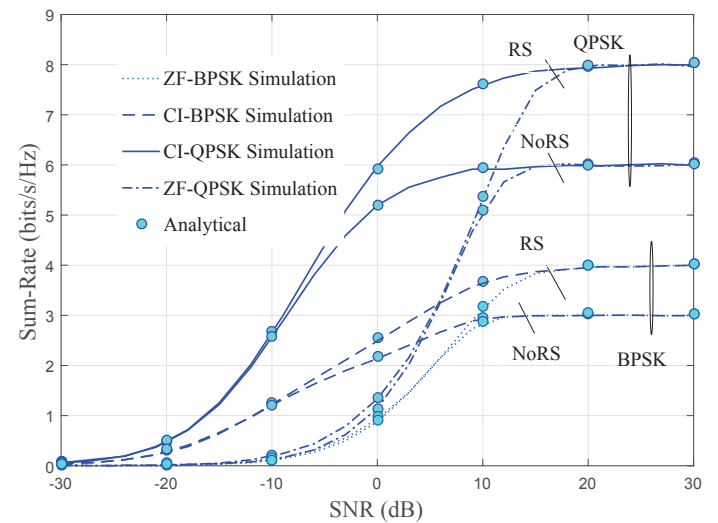

(a) Sum-rate versus SNR, when $d_{1}=d_{2}=d_{3}=1 \mathrm{~m}$.

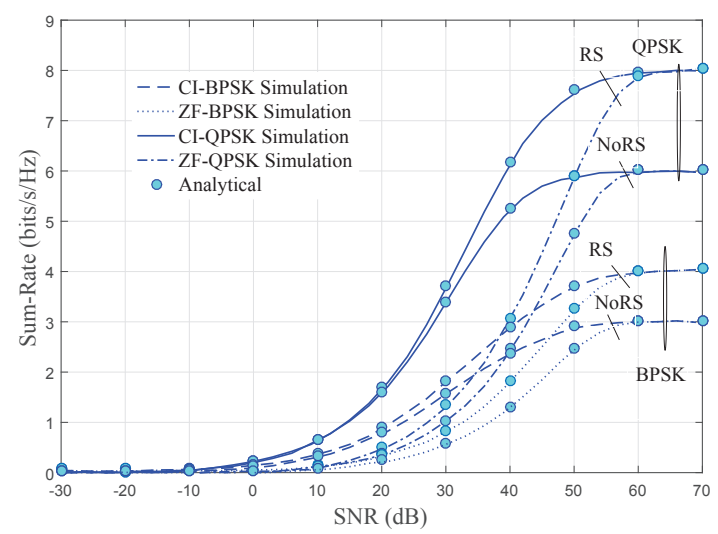

(b) Sum-rate versus SNR, when the users are randomly distributed.

Figure 2: Sum-rate versus SNR for RS and NoRS with different types of input, when $N=4$ and $K=3$.

sum rates. In addition, when the distance between the $\mathrm{BS}$ and the users increases the rate saturation occurs at high SNR values, due to larger path-loss. Furthermore, the gain attained by using RS with CI over the RS with ZF and NoRS does not depend on the users' locations.

Moreover, we investigate the impact of number of BS antennas and number of users on the system performance. Therefore, in Fig. 2 we plot the sum-rate versus the SNR, for the considered transmission schemes with BPSK, and QPSK, when $N=4$, and $K=3$. Fig. 2a presents the sum-rate when the distances are normalized to unit value, i.e, without the impact of the path-loss. Fig. $2 b$ shows the sum-rate when the users are uniformly distributed in a circle area of $60 \mathrm{~m}$ radius, where the BS is located at the center of this area and no user is closer the BS than $1 \mathrm{~m}$. Comparing the results in this figure with the results in Fig. 1, it is clear that increasing $K$ and/or $N$ results in enhancing the achievable sum-rate. In addition, comparing the sum rate achieved in Fig. 2a and Fig. 2b, we can see similar observations as in the case when $N=3, K=2$.

Finally, in order to clearly illustrate the impact of the power fraction $t$ on the system performance, we plot in Fig. 3 the sum-rate versus SNR for various values of $t$ with the CI precodnig under QPSK, when $N=3, K=2, d_{1}=1 \mathrm{~m}$ and $d_{2}=5 \mathrm{~m}$. Interestingly enough, it is noted that when $t$ is small, i.e., $t=0.1,0.5$, the sum-rate is at its lowest value 


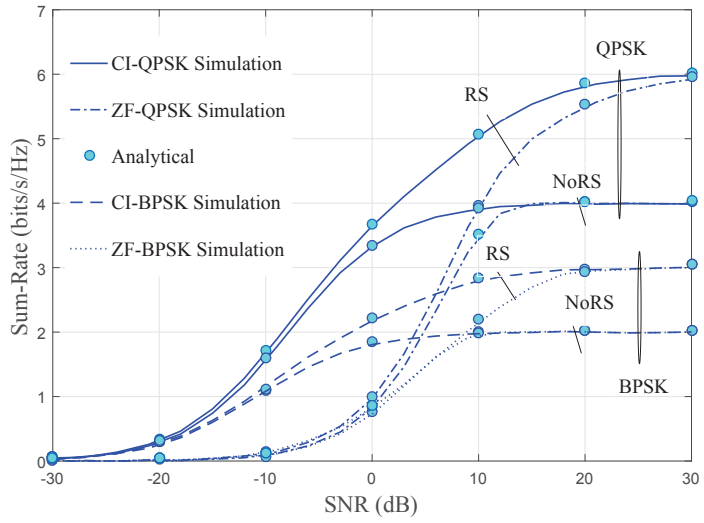

(a) Sum-rate versus SNR, when $d_{1}=d_{2}=1 \mathrm{~m}$.

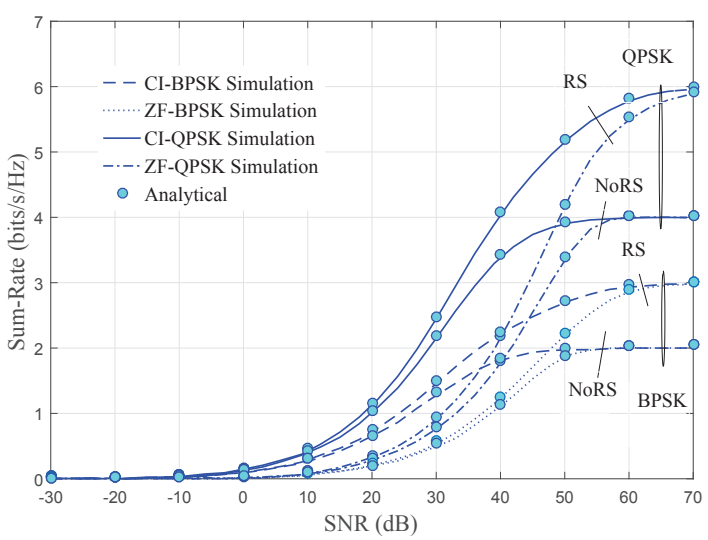

(b) Sum-rate versus SNR, when the users are randomly distributed.

Figure 1: Sum-rate versus SNR for RS and NoRS with different types of input, when $N=3$ and $K=2$.

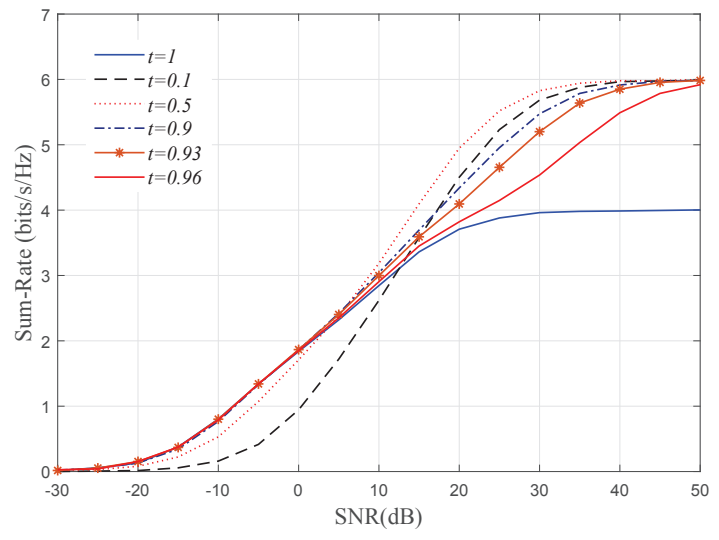

Figure 3: Sum-rate versus SNR for various values of $t$.

at low SNR, SNR $\leq 12 \mathrm{~dB}$, and at its highest value at high SNR, SNR $\geq 12 \mathrm{~dB}$. In addition, increasing $t$ leads to increase the sum rate at low SNR values and reduces the sum-rate at high SNR values. Therefore, it is better to select $t \approx 1$ at low SNR, which means the common part is unnecessary, and at high SNR vlaues the RS presents its superiority by selecting $t<1$ [2], [15].

\section{CONCLUSIONS}

In this paper we employed the CI precoding technique to enhance the sum-rate performed by RS scheme in MUMIMO systems under PSK input alphabet. New analytical expressions for the average sum-rate have been derived for, CI precoding technique, and ZF precoding technique in RS and NoRS scenarios. The results demonstrated that, RS with CI has greater sum-rate than RS with ZF and NoRS transmission techniques. In addition, increasing number of BS antennas and/ or number of users enhances the achievable sum-rate.

\section{REFERENCES}

[1] A. Salem and K. A. Hamdi, "Wireless power transfer in multi-pair two-way af relaying networks," IEEE Transactions on Communications, vol. 64, no. 11, pp. 4578-4591, Nov 2016.
[2] B. Clerckx, H. Joudeh, C. Hao, M. Dai, and B. Rassouli, "Rate splitting for mimo wireless networks: a promising phy-layer strategy for lte evolution," IEEE Communications Magazine, vol. 54, no. 5, pp. 98 105, May 2016.

[3] C. Hao, Y. Wu, and B. Clerckx, "Rate analysis of two-receiver miso broadcast channel with finite rate feedback: A rate-splitting approach," IEEE Transactions on Communications, vol. 63, no. 9, pp. 3232-3246, Sept 2015.

[4] H. Joudeh and B. Clerckx, "Robust transmission in downlink multiuser miso systems: A rate-splitting approach," IEEE Transactions on Signal Processing, vol. 64, no. 23, pp. 6227-6242, Dec 2016.

[5] A. Papazafeiropoulos and T. Ratnarajah, "Rate-splitting robustness in multi-pair massive mimo relay systems," IEEE Transactions on Wireless Communications, vol. 17, no. 8, pp. 5623-5636, Aug 2018.

[6] C. Masouros and E. Alsusa, "Dynamic linear precoding for the exploitation of known interference in mimo broadcast systems," IEEE Transactions on Wireless Communications, vol. 8, no. 3, pp. 1396-1404, March 2009.

[7] P. V. Amadori and C. Masouros, "Large scale antenna selection and precoding for interference exploitation," IEEE Transactions on Coтmunications, vol. 65, no. 10, pp. 4529-4542, Oct 2017.

[8] S. Timotheou, G. Zheng, C. Masouros, and I. Krikidis, "Exploiting constructive interference for simultaneous wireless information and power transfer in multiuser downlink systems," IEEE Journal on Selected Areas in Communications, vol. 34, no. 5, pp. 1772-1784, May 2016.

[9] A. Li and C. Masouros, "Interference exploitation precoding made practical: Optimal closed-form solutions for psk modulations," IEEE Transactions on Wireless Communications, pp. 1-1, 2018.

[10] R. J. Muirhead, Aspects of Multivariate Statistical Theory, 1982.

[11] W. Wu, K. Wang, W. Zeng, Z. Ding, and C. Xiao, "Cooperative multi-cell mimo downlink precoding with finite-alphabet inputs," IEEE Transactions on Communications, vol. 63, no. 3, pp. 766-779, March 2015.

[12] Y. Wu, C. Xiao, X. Gao, J. D. Matyjas, and Z. Ding, "Linear precoder design for mimo interference channels with finite-alphabet signaling," IEEE Transactions on Communications, vol. 61, no. 9, pp. 3766-3780, September 2013.

[13] Y. Wu, M. Wang, C. Xiao, Z. Ding, and X. Gao, "Linear precoding for mimo broadcast channels with finite-alphabet constraints," IEEE Transactions on Wireless Communications, vol. 11, no. 8, pp. 29062920, August 2012.

[14] M. Abramowitz and I. A. Stegun, Handbook of Mathematical Functions With Formulas, Graphs, and Mathematical Tabl, Washington,D.C.: U.S. Dept. Commerce, 1972

[15] M. Dai, B. Clerckx, D. Gesbert, and G. Caire, "A rate splitting strategy for massive mimo with imperfect csit," IEEE Transactions on Wireles. Communications, vol. 15, no. 7, pp. 4611-4624, July 2016. 